\title{
Utility of transient elastography in determining liver fibrosis
}

\author{
Authors \\ Deba Kumar Chakrabartty ${ }^{1}$, Bishnu Prasad Tripathy ${ }^{2 *}$ \\ Department of Radiology, Silchar Medical College, Assam, India \\ *Corresponding Author \\ Bishnu Prasad Tripathy
}

\begin{abstract}
Background:It was a descriptive study to evaluate the accuracy of acoustic radiation force impulse (ARFI) elastography for thedetection of liver fibrosis in patients with diffuse chronic liver diseases (CLD). Materials and Methods: In our study 36 adult healthy volunteers and 65 adult patients with Chronic liver diseases were evaluated using ARFI based US-elastography. Histopathological correlation was done as and when required.

Results: ARFI was done in all 36 healthy volunteers and all 65 CLD patients and valid measurements were obtained. The mean shear-wave velocity $(S W V)$ in healthy volunteers was $1.19 \pm 0.2 \mathrm{~m} / \mathrm{s}$. A gradual increase in mean SWV was noted from fibrosis of Grade F0 to F6 (Ishak's score) and a high positive correlation was found between the mean SWV on ARFI and fibrosis scores at liver biopsy (rho=0.8409). we found statistically significant difference $(p<0.001)$ between the mild (F1 and $F 2)$ and significant fibrosis (F3 and F4). The difference in the SWV measurements obtained from consecutive groups (i.e., F1 versus $F 2, F 2$ versus $F 3$, and F3 versus F4) was not statistically significant. Using the area under the receiver operating characteristic curve (AUROC), the best calculated cut-off SWVs for the presence of fibrosis $(\geq F 1)$, significant fibrosis $(\geq F 3)$, severe fibrosis $(\geq F 4)$, and cirrhosis (F6) were found to be 1.207, $1.347,1.513$, and $1.92 \mathrm{~m} / \mathrm{s}$, respectively. ARFI values were significantly higher in cirrhotic patients than in other patients $(p<0.001)$.

Conclusions: ARFI elastography can be used for non-invasive evaluation of liver stiffness and may help to differentiate between no/mild fibrosis and significant fibrosis. Hence when ARFI suggests significant fibrosis the need for liver biopsy may, indeed, be obviated.
\end{abstract}

\section{Introduction}

Liver fibrosis is a public health issue. The World Health Organization (WHO) defines cirrhosis as adiffuse process characterized by fibrosis and the conversion of normal liver architecture into structurally abnormal nodules. ${ }^{1}$ Alcoholism, Chronic hepatitis $\mathrm{B}$ and $\mathrm{C}$ commonly result in chronic liver disease (CLD) with liver fibrosis representing the organ's final response to injury. Over time, this process can result in cirrhosis, which can lead to the development of lifethreatening complications, such as portal hypertension, liver failure, and malignancy. The progression of CLD is determined fundamentally by the degree of fibrosis.

Although cirrhosis is traditionally considered as a state of irreversible fibrosis, accumulating evidence suggests that liver fibrosis could be reversible and that recovery from cirrhosis may be possible. Early treatment of the underlying disease 
may limit or even reverse the progression of fibrosis; hence, early detection of fibrosis is crucial for timely treatment. ${ }^{2}$

Currently, liver biopsy is considered as gold standard for evaluating the fibrosis in liver disease; however, it is limited by its invasive nature with associated risk of serious complications and sampling error and often it is notpossible to get a liver biopsy due to coagulopathy and/or thrombocytopenia., Repeated liver biopsies to assess the progression of liver disease are practicallyimpossible.The limitations and invasive nature of liver biopsy has spurred extensive interest in the development of non-invasive tests to measure liver fibrosis.

\section{Materials and methods}

Thirty-six adult healthy volunteers recruited from patient's relatives were evaluated. Sixty-five adult patients including 49 males and 16 females with a clinical suspicion of CLD and/or radiological diagnosis of cirrhosis based on real time ultrasound findings were included in this study during the period of July 2019 to June 2020. The procedure was explained to the participants, and written informed consent was obtained from all participants. The study was approved by Ethics committee.

The inclusion criteria for patients included $\rightarrow$ All patients referred for an ultrasound with a clinical diagnosis of having liver disease, ascites with suspected hepatic cause, chronic alcohol abuse and chronic viral hepatitis.

The exclusion criteria for patients included patients with diagnosed and/orsuspected malignant liver tumours, patients of splenectomy and patients who do not give consent.

Ultrasonographic elastography of the liver with ARFI using the Samsung RS80A S-shearwave system software was performed in all patients and healthy volunteers. In all patients, measurement was performed at the right liver lobe in segments 5,6 , and 8 , excluding vessels during suspended respiration. The number of successful measurements were aimed at 10 per patient with results expressed as propagation velocity in metres per second and kilopascal. ARFI liver elastography was only considered successful if 10 results could be obtained with a success rate of $>60 \%$. Furthermore, the mean value of successful measurements was considered representative of liver stiffness in a given patient only if the interquartile range (IQR) of all successful measurements was $<30 \%$ of the mean value. Thus, ARFI results were only considered valid if the success rate was above $60 \%$ and the IQR $<30 \%$ of the mean values. The equipment listed the shearwave velocity (SWV) in the region of interest as well as the depth at which the measurement was performed.

\section{Liver Biopsy}

Liver biopsy was obtained in all patients and liver fibrosis stages were evaluated according to the Ishak scoring system using a seven-point (F0-F6) scale by a pathologist. Biopsies were performed within 7 days following the elastographic evaluation.

\section{Statistical Analysis}

Results obtained at ultrasound elastography were compared with those of histopathology using the software SPSS (version 27.0; SPSS, Chicago, IL, USA). All data were presented as mean \pm 2 SD. $\mathrm{p}<0.05$ was considered statistically significant. Continuous variables of two independent groups were compared with Student's t-test. Correlations between parameters were expressed with Pearson's or two tailed pairwise correlation coefficients, as appropriate. The area under the curve (AUC) was estimated for continuous variables using receiver operating characteristic (ROC)analysis.

\section{Results}

In our study ARFI were measured in thirty-six adult healthy volunteers and in sixty-five adult patients. Valid measurements (IQR $<30 \%$ ) were obtained. Mean age of healthy volunteers and patients were 48.7 years and 51.9 years. In both 
healthy volunteers and patient groups $75 \%$ were male (Table 1). The cause of chronic liver disease was HBV (24.5), HCV (33.8), Alcoholism (38.5\%) and combined $\mathrm{HBV}+\mathrm{HCV}$ (3.1\%) infection (Fig: - 1).
Table-1

\begin{tabular}{|c|c|c|c|}
\cline { 2 - 4 } \multicolumn{1}{c|}{} & Male & Female & Total \\
\hline $\begin{array}{c}\text { Healthy } \\
\text { volunteer }\end{array}$ & 28 & 8 & 36 \\
\hline Patient & 49 & 16 & 65 \\
\hline
\end{tabular}

Figure 1: Distribution of cases according to etiology

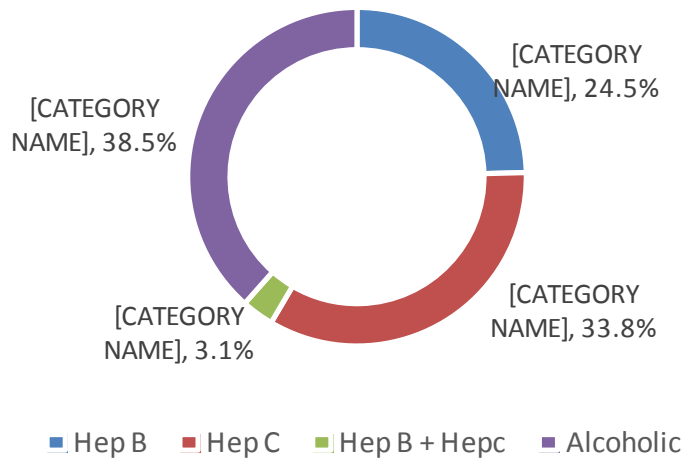

\section{ARFI Measurements}

\section{Healthy Volunteers}

The range of SWVs in normal healthy volunteers wasbetween $0.905-1.314 \mathrm{~m} / \mathrm{s}$ with a mean SWV of $1.119 \pm 0.20 \mathrm{~m} / \mathrm{s}$. Nosignificant differences were found between the mean ARFIvalues in men versus women $(1.119 \pm 0.19$ versus $1.12 \pm 0.24 \mathrm{~m} / \mathrm{s})$ and also among different age groups ofhealthy volunteers (Pearson's correlation coefficient rho $=0.188$ for age and ARFI values).

\section{Patient Group}

The mean SWVs of the various patient groups accordingto grade of fibrosis are tabulated in Table 2. ARFI liverpropagation velocity was positively correlated to histologywith Spearman's correlation coefficient rho $=0.840 \quad(\mathrm{p}<0.0001)$. Thus, the mean SWV showed an increasingtrend with increasing grade of fibrosis; however, there wasan overlap in the absolute SWV between patients and thevolunteer group and between various grades of fibrosis. By using thearea under the ROC curve (AUROC) and applying an SWVcut-off of $1.347 \mathrm{~m} / \mathrm{s}$ for detection of significant fibrosis $(\geq F 3)$, the sensitivity, specificity, positive predictive value(PPV), and negative predictive value (NPV) were $91.3 \%, 83.7 \%, 75 \%$, and $95 \%$, respectively. Similarly, a cut-off ofl. $92 \mathrm{~m} / \mathrm{s}$ for the detection of cirrhosis (F5 and F6) resulted in a sensitivity of $90.9 \%$, specificity of $94.4 \%$, PPV of $77 \%$, and NPV of $98 \%$ respectively.

Table 2

\begin{tabular}{|l|c|c|c|}
\hline Ishak's grade & No. of patients (N) & $\begin{array}{c}\text { Mean } \\
\text { SWV(m/s) } \pm 2 S D\end{array}$ & Range (min-max) \\
\cline { 1 - 1 } Healthy volunteers & 36 & $1.119 \pm 0.2$ & $0.905-1.314$ \\
\hline Patients & 13 & $1.153 \pm 0.24$ & $0.96-1.438$ \\
\hline F0 & 21 & $1.253 \pm 0.18$ & $1.105-1.509$ \\
\hline F1 & 8 & $1.327 \pm 0.2$ & $1.158-1.491$ \\
\hline F2 & 6 & $1.413 \pm 0.71$ & $0.879-1.891$ \\
\hline F3 & 5 & $1.861 \pm 0.76$ & $1.389-2.388$ \\
\hline F4 & 1 & 2.041 & - \\
\hline F5 & 11 & $2.85 \pm 1.35$ & $1.890-4.310$ \\
\hline
\end{tabular}




\section{Discussion}

There are various studies comparing noninvasivemethods of evaluationin CLD with liver biopsy to assess whether they merit replacing this invasive method in the future. Non-invasive tests are safe, easy-to-perform, and are thus potentially useful for large-scale screening of liver fibrosis in patients with diffuse CLD.

Thirty-six healthy volunteers and sixty-five patients with a clinical and ultrasonographic suspicion of chronic liver disease due to hepatitis $\mathrm{B}$, hepatitis $\mathrm{C}$, and alcoholism referred from the Department of Medicine, Surgery, and OBGY of Silchar medical college were included in the study with the aim to evaluate patients of chronic liver disease with conventional ultrasonography and to obtain ARFI (Acoustic Radiation Force Impulse) elastography. Thesemeasurements were compared with those of normal healthy volunteers.

\section{Healthy volunteers}

In our study, the healthy volunteer group consisted of 28 males and 8 females in a ratio of $3: 1$. The maximum numbers $(39.2 \%)$ of volunteers were in 41-50 years age group with a mean age of 48.7 years. Alina Popescu et al evaluated 82 healthy volunteers without known liver pathology including 50 women and 32 men with a mean age of $35.3 \pm 14.8$ years. ${ }^{5}$

Andrew T. Trout et al evaluated 32 adult healthy volunteers with median age of 48.92 years without known liver disease. ${ }^{6}$

All the healthy volunteers in our study had normal greyscale ultrasound. The range of Shear Wave Velocity (SWV) in normal healthy volunteers on ARFI ranged between 0.905 to $1.314 \mathrm{~m} / \mathrm{s}$ with a mean SWV of $1.119 \pm 0.2 \mathrm{~m} / \mathrm{s}$. Only $8 / 36(22.2 \%)$ healthy volunteers had $\mathrm{SWV}>1.2 \mathrm{~m} / \mathrm{s}$. No significant difference was seen in ARFI values amongst healthy males and healthy females $(1.119 \pm 0.19 \mathrm{~m} / \mathrm{s} \quad$ vs $1.12 \pm 0.22 \mathrm{~m} / \mathrm{s}) \quad(\mathrm{p}$ value $=0.2911$ ) and among various age groups $(\mathrm{rho}=-0.702$, negative correlation). Our findings are similar to Andrew T. Trout et al who found that mean value of normal ARFI measurements
$1.32 \pm 0.13 \mathrm{~m} / \mathrm{s} .{ }^{6}$ Our findings are also similar to Alina Popescu et al who found the mean value of ARFI measurements in normal individuals to be $1.15 \pm 0.21 \mathrm{~m} / \mathrm{s}$ (range $0.69-1.60 \mathrm{~m} / \mathrm{s}$ ) with only (18.4\%) having ARFI value higher than the mean value of $1.15 \pm 0.21 \mathrm{~m} / \mathrm{s}$. They found no significant difference between the mean ARFI values in men and women $(1.16 \pm 0.21$ vs. $1.14 \pm 0.22 \mathrm{~m} / \mathrm{s}, \mathrm{p}=0.67)$, and among different age groups $(p>0.05) .{ }^{5}$ Our findings parallel those ofHorster et al who obtained a mean ARFI set of $1.19 \mathrm{~m} / \mathrm{s}$ (range 0.77-1.63) in Caucasian volunteers and demonstrated that age and gender did not influence the SWV. ${ }^{7}$ Our results are also parallel to several other studies as well like Rifai K et al who found no statistical correlation between ARFI elastography measurements and patient's age, sex, or BMI (all p > 0.05). ${ }^{8}$ Kim et al included in their series 133 subjects with normal liver and obtained the mean SWV (or the normal liver group) as $1.08 \pm 0.15 \mathrm{~m} / \mathrm{s}$, Friedrich-Rust et al included 20 healthy adult volunteers as a reference group and obtained a mean SWV of $1.13 \pm 0.23 \mathrm{~m} / \mathrm{sec}$ (range 0.85-1.42 m/sec). ${ }^{9,10}$ A negative correlation between shear wave velocity and the variables like age, sex, and BMI was also found by Florentina Guzmñn-A roca et al. ${ }^{11}$ Our study correlates well with the above-mentioned studies. Our study is at slight variance with that of Gallotti et al who obtained a mean value of $1.59 \mathrm{~m} / \mathrm{s}$, higher than the values from the other studies. ${ }^{12}$

\section{Patient group}

The patient group consisted of 49 male and 16 female patients in a ratio of approximately $3: 1$. All the patients were between 20 to85 years with a meanage of 51.9 years. The majorityof the patients $(30 \%)$ were in the range of 50-60 years of age group. The largest subgroup of patients seen in our study were alcoholics (38.5\%), while $33.8 \%$ were $\mathrm{HCV}$ positive cases, $24.5 \%$ were $\mathrm{HBV}$ positive and $3.1 \%$ were positive for both $\mathrm{HBV}$ and HCV markers. 


\section{ARFI}

In our study, the ARFI shear wave velocities in patients ranged between $0.96-4.310 \mathrm{~m} / \mathrm{s}$. When the velocities were analysed according to the grades of fibrosis based on the Ishak's fibrosis scores; patients with F0 fibrosis had SWV ranges from $0.96-1.438 \mathrm{~m} / \mathrm{s}$ with a mean of $1.153 \pm 0.24 \mathrm{~m} / \mathrm{s}$. Similarly, the patients with F1 had a range between $1.105-1.509 \mathrm{~m} / \mathrm{s}$ with a mean of $1.253 \pm 0.18 \mathrm{~m} / \mathrm{s}$, with $\mathrm{F} 2$ had a range between $1.158-1.491 \mathrm{~m} / \mathrm{s}$ with a mean of $1.327 \pm 0.20 \mathrm{~m} / \mathrm{s}$. with F3 had SWV range between $0.879-1.891 \mathrm{~m} / \mathrm{s}$ with a mean of $1.413 \pm 0.71 \mathrm{~m} / \mathrm{s}$, with F4 had SWV range between $1.389-2.388 \mathrm{~m} / \mathrm{s}$ with a mean of $1.861 \pm 0.76 \mathrm{~m} / \mathrm{s}$. The only patient with F5 fibrosis had SWV of $2.041 \mathrm{~m} / \mathrm{s}$ and the mean SWV was $2.85 \pm 1.36 \mathrm{~m} / \mathrm{s}$ (range $1.890-4.310 \mathrm{~m} / \mathrm{s}$ ) for the patients with F6 fibrosis. Thus, there was an overlap in the ranges of SWV but a gradual increase in mean shear wave velocities was noted from F0 to F6. The SWV on ARFI and fibrosis scores were found to be positively correlated $(\mathrm{rho}=0.8409)$. These findings are in agreement with several other studies. Mireen FriedrichRust who measured the Shear Wave velocities in 86 patients with velocities ranging from 0.84 to $3.83 \mathrm{~m} / \mathrm{sec}$. According to the different Metavir fibrosis scores, the mean SWV was $1.16 \pm 0.17 \mathrm{~m} / \mathrm{s}$ for patients with stage $F 0,1.18 \pm 0.18 \mathrm{~m} / \mathrm{s}$ for patients with stageF1, $1.34 \pm 0.34 \mathrm{~m} / \mathrm{s}$ for patients with stage $F 2,1.75 \pm 0.51 \mathrm{~m} / \mathrm{s}$ for patients with stage $\mathrm{F} 3$, and $2.38 \pm 0.74 \mathrm{~m} / \mathrm{s}$ for patients with stage F4. Spearman correlation coefficients for the correlations between fibrosis stage and the results of ARFI imaging indicated significant correlations $(r h o=0.71)$ who also documented an increase in mean SWV. ${ }^{9}$ Lupsor et al who also reported a similar correlation (spearman's correlation coefficient $=0.717$ ) between histological grades of fibrosis. The mean SWV values in various stages of fibrosis were; $1.079 \pm 0.150 \mathrm{~m} / \mathrm{s} \quad$ (F0-F1), $1.504 \pm 0.895 \mathrm{~m} / \mathrm{s} \quad(\mathrm{F} 2), \quad 1.520 \pm 0.575 \mathrm{~m} / \mathrm{s} \quad(\mathrm{F} 3)$, $2.552 \pm 0.782 \mathrm{~m} / \mathrm{s}(\mathrm{F} 4)$. Our values are similar to the above-mentioned studies although absolutest age-wise comparison cannot be made due to different fibrosis staging systems. ${ }^{13} \mathrm{~A}$ direct, strong, correlation between ARF Imeasurements and fibrosis was also demonst rated by Sporea et $\mathbf{a l}^{14,15}$ in two separate studies and Mazhar Hague et al $^{16}$ although Fie rbinteanu-B raticevici $\mathbf{C}$ et $\mathbf{a l}^{17}$ found a slightly higher correlation coefficient of 0.919 and between ARFI and fibrosis.

\section{Comparison of ARFI measurements between} healthy volunteers and patients of chronic liver disease

In our study, although we did not find any significant difference between the mean SWV of healthy volunteers $(1.119 \pm 0.20 \mathrm{~m} / \mathrm{s})$ and patients with F0 fibrosis $(1.153 \pm 0.24 \mathrm{~m} / \mathrm{s})(\mathrm{p}=0.4969)$, we did find a significant difference betweent heme an SWV amongst healthy volunteers $(1.119 \pm 0.20 \mathrm{~m} / \mathrm{s})$ and the patient group considered as a whole (F0 to cirrhosis groups) (mean SWV-1.587 $\pm 1.36 \mathrm{~m} / \mathrm{s}$ ) (p $<0.0001)$. An overlap in the mean SWV was however present in the two groups. These results parallel those of Mireen Friedrich-R ust who documented that the healthy volunteers had a mean ARFI velocity comparable to that of the patients with Metavir fibrosis stage F0, individual measurements in the healthy volunteers ranged from the velocities documented in patients without histologic fibrosis to the velocities measured in patients with liver cirrhosis. $^{9}$

However, in our study, the mean SWV in the F1 group at $1.253 \mathrm{~m} / \mathrm{s}$ was higher than the volunteer's group $(1.119 \mathrm{~m} / \mathrm{s})$ and the difference was statistically significant $(\mathrm{p}<0.0001)$. Similarly, though overlapping values were noted, the difference in mean SWV was statistically significant $(\mathrm{p}<0.0001)$ between healthy volunteers and mild fibrosis F1-F2 group $(1.274 \mathrm{~m} / \mathrm{s})$ and between healthy volunteers and significant fibrosis $(\geq F$ 3-Ishak's) group at mean $\mathrm{SWV}$ of $2.227 \mathrm{~m} / \mathrm{s}(\mathrm{p}<0.0001)$.

$V$ Jain et al demonstrated that no significant difference $(p=0.35)$ between the mean SWV of healthy volunteers and patients with F0 fibrosis with an overlap in the mean SWV in the two groups. They also demonstrated that mean SWV 
was statistically significant $(\mathrm{p}<0.0001)$ between healthy volunteers and mild fibrosis (Ishak's F1F2), between healthy volunteers and significant fibrosis (Ishak's $\geq$ F3 Ishak's). ${ }^{18}$ Golo Petzold et al demonstrated that liver stiffness in the liver patient cohort was not significantly different from that in the healthy-volunteer cohort $(\mathrm{p}=0.13)$. there was no overlap of liver stiffness measurements between the patients without liver disease and the cirrhosis cohort $(\mathrm{p}<0.001) .{ }^{19}$

\section{Comparison of ARFI measurements between various groups of fibrosis}

We did not find any significant difference between the mean SWV of F1 versus F2 fibrosis groups $(\mathrm{p}=0.071), \mathrm{F} 2$ versus $\mathrm{F} 3(\mathrm{p}=0.5186)$, and $\mathrm{F} 3$ versus F4 groups $(\mathrm{p}=0.06)$ (Ishak's). Lupsor et al also demonstrateda certain degree of overlap between the consecutive stages F1-F2 ( $\mathrm{p}=0.072)$ and F2-F3 ( $\mathrm{p}=0.965) .{ }^{13}$ Similarly, Fie rbinteanuBraticevici $\mathbf{C}$ et al observed an overlap between F0-F1 and F2 and stated that the increase in liver propagation velocity was more important between stages F2 $(1.21 \mathrm{~m} / \mathrm{s})$ and $\mathrm{F} 3$ than between stages $\mathrm{F} 1$ and F2. ${ }^{17} \mathrm{~V}$ Jain et al demonstrated that no significant difference between the mean SWV of healthy volunteers and patients with F0 fibrosis with an overlap in the mean SWV in the two groups. Similarly, though, overlapping values were noted, the difference in mean SWV was statistically significant $(\mathrm{p}<0.0001)$ between healthy volunteers and mild fibrosis F1-F2, between healthy volunteers and significant fibrosis ( $\geq$ F3 Ishak's) group $(p<0.0001){ }^{18}$

When the mean SWV in the no fibrosis group (F0) patients was compared with the mild fibrosis group (i.e., F1-F2), the values were found to be significantly higher in the mild fibrosis group than no fibrosis group (F0) (1.274 vs $1.153 \mathrm{~m} / \mathrm{s})$, $\mathrm{p}=0.0013$. Similarly, the mean SWV of cirrhotic (F5-6) groups was significantly higher than those of the non-cirrhotic fibrosis group (F1-F4) (2.78 vs $1.22 \mathrm{~m} / \mathrm{s}) ; \mathrm{p}<0.0001$.

\section{Sensitivity and Specificity}

By using area under ROC applying SWV cut off of $1.207 \mathrm{~m} / \mathrm{s}$ for the presence of fibrosis $(\geq F 1)$ we obtain a sensitivity of $80.8 \%$, specificity of $76.9 \%$, and PPV and NPV of $93 \%$ and $50 \%$ respectively. Similarly, a cut-off of $1.347 \mathrm{~m} / \mathrm{s}$ for detection of significant fibrosis $(\geq \mathrm{F} 3)$ yielded a sensitivity, specificity, PPV, and NPV as 91.3\%, 83.7\%, 75\%, and $95 \%$ respectively. A cut-off of $1.513 \mathrm{~m} / \mathrm{s}$ for detection of severe fibrosis $(\geq F 4)$ resulted in a sensitivity of $94.1 \%$, specificity of $95.8 \%$, PPV of $89 \%$, and NPV of $98 \%$ respectively. Again, a cutoff of $1.920 \mathrm{~m} / \mathrm{s}$ for detection of cirrhosis (F6) resulted in a sensitivity of $90.9 \%$, specificity of $94.4 \%$. PPV of $77 \%$ and NPV of $98 \%$ respectively. The cut-off values are quite similar to those reported by Sporea et $\mathbf{a l}^{15}$ who also had compared ARFI with liver biopsy (LB) and determined the cut-off values for predicting the presence of fibrosis ( $\mathrm{F} \geq 1$ Metavir), significant fibrosis $(F \geq 2)$, severe fibrosis $(F \geq 3)$ and cirrhosis $(\mathrm{F}=4)$, as 1.19, 1.21, 1.58 and $1.82 \mathrm{~m} / \mathrm{s}$ respectively which were quite similar to our study results. Liver stiffness measurements had $73 \%$, $84 \%, 84 \%$ and $91 \%$ Sensitivity respectively: $93 \%$. $91 \%, 94 \% .90 \%$ Specificity, respectively. In the study by Lupsor et al ${ }^{13}$ the cut-off values $(\mathrm{m} / \mathrm{s})$ predictive for each stage of fibrosis were: $1.19 \mathrm{~m} / \mathrm{s}$ $(\mathrm{F} \geq 1), 1.34 \mathrm{~m} / \mathrm{s} \quad(\mathrm{F} \geq 2), 1.61 \mathrm{~m} / \mathrm{s}(\mathrm{F} \geq 3)$ and $2.00 \mathrm{~m} / \mathrm{s}$ (F4) with sensitivity and specificity were $(62.07 \%$, $67.80 \%, 79.07,80.0 \%)$ and $(85.71 \%, 92.86 \%$, $94.83 \%$, 95.45\%) respectively. Fierbinteanu-

Braticevici et al demonstrated that ARFI elastography could predict fibrosis of METAVIR stage $\geq$ F2 with a validity of $90.2 \%$. They calculated that optimal cut-off between stages F1 and $\mathrm{F} 2$ to be $1.21 \mathrm{~m} / \mathrm{s}$. At this value, ARFI elastography had a sensitivity of $89.4 \%$ and a specificity of $100 \%$. They also found that ARFI can predict even better F3 and F4 stage fibrosis. The optimal cut-off value to identify fibrosis stage F3 or higher was $1.54 \mathrm{~m} / \mathrm{s}$, with sensitivity and specificity of $97 \%$ and $100 \%$ respectively. The optimal cut-off value in predicting cirrhosis (stage 
F4) was $1.94 \mathrm{~m} / \mathrm{s}$ with a sensitivity of $100 \%$ and a specificity of $98.1 \% .^{17}$

\section{Conclusion}

Chronic parenchymal liver disease (CLD) is a common presenting condition in a medical institution. Alcoholism and viral hepatitis are common causes of CLD. Imaging helps in early diagnosis, or exclude the presence of any structural lesion, and thus helps to guide further management.

Among the imaging modalities, ultrasonography based elastography is highly accurate for detecting and staging hepatic fibrosis.

ARFI elastography is a non-invasive, reliable, and repeatable diagnostic test for grading of liver fibrosis. It performs better than the biochemical indices to differentiate between severe fibrosis and cirrhosis of the liver. ARFI elastography may also obviate need for FNABC in selected patients.

\section{Bibliography}

1. Anthony, P. P. et al. The morphology of cirrhosis. Recommendations on definition, nomenclature, and classification by a working group sponsored by the World Health Organization. J. Clin. Pathol.31, 395-414 (1978).

2. Sun, Y. et al. New classification of liver biopsy assessment for fibrosis in chronic hepatitis B patients before and after treatment. Hepatol. Baltim. Md65, 14381450 (2017).

3. Poniachik, J. et al. The role of laparoscopy in the diagnosis of cirrhosis. Gastrointest. Endosc.43, 568-571 (1996).

4. Cadranel, J. F., Rufat, P. \& Degos, F. Practices of liver biopsy in France: results of a prospective nationwide survey. For the Group of Epidemiology of the French Association for the Study of the Liver (AFEF). Hepatol. Baltim. Md32, 477-481 (2000).

5. Popescu, A. et al. The mean values of liver stiffness assessed by Acoustic Radiation
Force Impulse elastography in normal subjects. Med. Ultrason.13, 33-37 (2011).

6. Trout, A. T., Xanthakos, S. A., Bennett, P. S. \& Dillman, J. R. Liver Shear Wave Speed and Other Quantitative Ultrasound Measures of Liver Parenchyma: Prospective Evaluation in Healthy Children and Adults. Am. J. Roentgenol.214, 557-565 (2020).

7. Horster, S., Mandel, P., Zachoval, R. \& Clevert, D. A. Comparing acoustic radiation force impulse imaging to transient elastography to assess liver stiffness in healthy volunteers with and without valsalva manoeuvre. Clin. Hemorheol. Microcirc.46, 159-168 (2010).

8. Rifai, K. et al. Clinical feasibility of liver elastography by acoustic radiation force impulse imaging (ARFI). Dig. Liver Dis. Off. J. Ital. Soc. Gastroenterol. Ital. Assoc. Study Liver43, 491-497 (2011).

9. Friedrich-Rust, M. et al. Liver fibrosis in viral hepatitis: noninvasive assessment with acoustic radiation force impulse imaging versus transient elastography. Radiology252, 595-604 (2009).

10. Kim, J. E. et al. Acoustic radiation force impulse elastography for chronic liver disease: comparison with ultrasound-based scores of experienced radiologists, ChildPugh scores and liver function tests. Ultrasound Med. Biol.36, 1637-1643 (2010).

11. Guzmán-Aroca, F. et al. Reproducibility of shear wave velocity measurements by acoustic radiation force impulse imaging of the liver: a study in healthy volunteers. J. Ultrasound Med. Off. J. Am. Inst. Ultrasound Med.30, 975-979 (2011).

12. Gallotti, A., D’Onofrio, M. \& Pozzi Mucelli, R. Acoustic Radiation Force Impulse (ARFI) technique in ultrasound with Virtual Touch tissue quantification of 
the upper abdomen. Radiol. Med. (Torino)115, 889-897 (2010).

13. Lupsor, M. et al. Performance of a new elastographic method (ARFI technology) compared to unidimensional transient elastography in the noninvasive assessment of chronic hepatitis C. Preliminary results. J. Gastrointest. Liver Dis. JGLD18, 303-310 (2009).

14. Sporea, I. et al. How efficient is acoustic radiation force impulse elastography for the evaluation of liver stiffness? Hepat. Mon.11, 532-538 (2011).

15. Sporea, I. et al. Is ARFI elastography reliable for predicting fibrosis severity in chronic HCV hepatitis? World J. Radiol.3, 188-193 (2011).

16. Haque, M., Robinson, C., Owen, D., Yoshida, E. M. \& Harris, A. Comparison of acoustic radiation force impulse imaging (ARFI) to liver biopsy histologic scores in the evaluation of chronic liver disease: A pilot study. Ann. Hepatol.9, 289-293 (2010).

17. Fierbinteanu-Braticevici, C. et al. Acoustic radiation force imaging sonoelastography for noninvasive staging of liver fibrosis. World J. Gastroenterol. WJG15, 55255532 (2009).

18. Jain, V., Dixit, R., Chowdhury, V., Puri, A. S. \& Gondal, R. Can acoustic radiation force impulse elastography be a substitute for liver biopsy in predicting liver fibrosis? Clin. Radiol.71, 869-875 (2016).

19. Petzold, G., Hofer, J., Ellenrieder, V., Neesse, A. \& Kunsch, S. Liver Stiffness Measured by 2-Dimensional Shear Wave Elastography: Prospective Evaluation of Healthy Volunteers and Patients With Liver Cirrhosis. J. Ultrasound Med. Off. J. Am. Inst. Ultrasound Med.38, 1769-1777 (2019). 